\title{
When individual differences meet society: on the complex relationships between boredom proneness, material deprivation, and aspects of subjective well-being among young adolescents
}

\begin{abstract}
Even though boredom has been shown to be a distinct emotional experience that is prevalent among youths and that predicts a wide range of personal and societal problems, it remains often overlooked and poorly understood. Therefore, based on the Flemish data from the 2018 edition of the International Survey of Children's Well-Being, this article studies boredom proneness among young adolescents (average age: 12). Confirmatory factor analysis showed that, the short version of the Boredom Proneness Scale - Child Version contains two dimensions referring to internal - a difficulty in keeping oneself interested and entertained and external stimulation - the need for change and variety in the situations one encounters. Subsequent regression analyses demonstrated that both boredom proneness and social conditions predict aspects of subjective well-being (i.e., leisure boredom and life satisfaction). Our analyses revealed complex interactions between individual differences and social conditions with respect to aspects of well-being.
\end{abstract}

Keywords: boredom, boredom proneness, young adolescents, child-reported material deprivation, ISCWeB 


\section{Introduction}

Boredom refers to an "aversive state of wanting, but being unable, to engage in satisfying activity" (Eastwoord, Frischen, Fenske \& Smilek, 2012: 483). Although boredom relates to other mental states such as depression, apathy and anhedonia (the inability to feel pleasure), both on conceptual/theoretical (Farmer \& Sundberg, 1986) and empirical grounds (Goldberg, Eastwood, Laguardia \& Danckert, 2011), it has been shown that boredom is a distinct emotional experience rather than a secondary symptom to other psychological states. Boredom is also prevalent among youths. Recent research indicates that approximately $20 \%$ of 8 th and 10 th graders in the USA suffer from large amounts of boredom (Martz, Schulenberg, Patrick \& Kloska, 2018). Finally, boredom is consequential. Indeed, boredom has been shown to predict a wide range of personal and societal problems (Spaeth, Weichod \& Silbereisen, 2015). Notwithstanding all of this, relatively little research has studied boredom among young adolescents and children. Indeed, boredom is described as an "overlooked phenomenon" (Pekrun, Hall, Goetz \& Perry, 2014) which is "poorly understood" (Eastwood, Frischen, Fenske \& Smilek, 2012: 483).

Boredom is thought to result from (the combination of) two types of factors: (1) people's predisposition to be bored - boredom as a trait: some people are more easily bored than others - and (2) the amount of arousal circumstances evoke - state boredom: some situations are more boring than others. Each factor has led to a separate strand of research. The first directs attention to individual differences in what has been called boredom proneness (BPS) (Goldberg et al., 2011; Lehr \& Todman, 2009; Sommers \& Vodanovich, 2000). The second motivated scholars to study social differences in boredom and showed, for example, that people who live in deprived situations suffer more from (leisure) boredom (Martz et al., 2018; Spruyt, Vandenbossche, Keppens, Siongers \& Van Droogenbroeck, 2018). 
An important limitation of the current literature on boredom is that both strands of research have largely developed independently from each other. First, research on boredom proneness has invested much effort into scale development and studying the outcomes of BPS, but did so by relying on convenience and/or clinical samples among highly specific target groups (e.g., people who suffer from extreme deprivation). Typically, such samples lack social heterogeneity, thus leaving unanswered the question as to how widespread boredom is and which social differences are present in boredom. Moreover, except from a few studies (Lehr \& Todman, 2009), boredom scales have not been systematically tested among young adolescents and children. One objective of this paper is to fill that gap. Second, research that relied on large representative samples often have no measures of boredom proneness at their disposal (Martz et al., 2018; Spruyt et al., 2018). This raises the question whether observed social differences in (leisure) boredom and its consequences are really socially-induced rather than the result of individual traits. To answer that question, one should study social differences while simultaneously taking into account individual differences (i.e., boredom proneness). This is important because depending on the outcome of such analyses, different strategies to remedy boredom should be considered.

Against that background this paper seeks to answer two questions: (1) Can we measure boredom proneness among (a socially heterogeneous group of) young adolescents? And (2) How does the predictive power of boredom proneness relates/compares to characteristics of these young adolescents' social position and life circumstances for leisure boredom and life satisfaction?

To answer our research questions we used data from the Flemish edition of the International Survey of Children's Well-Being (ISCWeB) gathered in 2018 among a large probability sample of Flemish young adolescents in the last grade of primary school $(N=988)$. Confirmatory Factor Analysis was used to assess the scale properties of the short version of the Boredom Proneness Scale - Child Version (BPS-C) as proposed by Lehr and Todman (2009). Next, regression 
analysis was used to assess social differences in (1) leisure boredom and (2) adolescents' general life satisfaction after taking into account individual differences in boredom proneness. This study is one of the first that is able to assess the relative explanatory power and potential interplay between factors that consider a young person's psychological traits (BPS-C) and features of their personal living conditions with respect to potential outcomes (i.e., material deprivation).

\section{Boredom proneness}

Boredom proneness refers to an individual's tendency to experience boredom. A boredom prone person is someone who "(a) lacks motivation, goals, ambition, and a sense of meaning or purpose; (b) experiences varying degrees of negative affect, such as hopelessness, anxiety, depression, hostility and loneliness; and (c) engages in maladaptive and unhealthy behaviors" (Watt \& Vodanovich, 1999: 311). Boredom proneness refers to the observation that people differ in the extent to which they perceive situations (monotonous vs. exciting), the level of arousal they need, know what they desire, and their ability to concentrate. Boredom proneness has been shown to predict a wide range of outcomes (Vodanovich \& Watt, 2016), such as binge drinking, internet addiction, less engagement in hobbies and sport activities (Biolcati, Mancini \& Trombini, 2018).

Different scales have been proposed to measure boredom proneness (Vodanovich, 2003). One of the most frequently used, and the one we rely on in our empirical analysis, concerns the Boredom Proneness Scale (BPS). This measure, developed by Farmer and Sundberg (1986: 10), consists of 28 items that tap into “one's connectedness with one's environment on different situational dimensions, as well as the ability to access adaptive resources and realize competencies". Boredom proneness is a multidimensional concept but no consensus has been reached concerning how many subdimensions (ranging from 2 to 7 ) the original 28-items comprise exactly (Vodanovich \& Watt, 2016). Two dimensions, however, have consistently 
emerged, namely one's inability to generate interesting activities for oneself or a low selfregulation (i.e., internal stimulation) and people's inclination to perceive low environmental stimulation or a need for variety and change (i.e., external stimulation). The internal stimulation dimension refers to difficulties in keeping oneself interested and entertained (example item: I find it easy to entertain myself) and has been shown to be related to a need for cognition (Watt \& Blanchard, 1994). External stimulation refers to the need for excitement, change and variety and the inability to engage in satisfying activities (example item: Many things I have to do are repetitive and monotonous). So, whereas the internal stimulation factor represents a rather apathetic state of ennui, the external stimulation dimension was formulated to reflect a tendency to become agitated when an individual is motivated but fails to engage in their environment.

The observation that two dimensions have consistently emerged inspired Vodanovich and colleagues (2005) to propose a 12-item short version of the boredom proneness (BPS-SF) that covers both crucial dimensions of boredom proneness. Although even for the BPS-SF the discussion concerning the exact factor structure has not been settled (Vodanovich \& Watt, 2016), there seems to be sufficient empirical support for the idea that this 12 -item version of the BPS covers the most important aspects of individuals' boredom proneness. Lehr and Todman (2009) adapted the BPS measure to study boredom proneness in children aged 8 years (BPS-C) in which item wordings were slightly rephrased to fit the target group. To date, this remains the only study which studied boredom proneness among children. Our study aims to contribute to the further development of this research area.

\section{The explanatory power of individual versus social differences regarding leisure boredom} and life satisfaction

Boredom proneness and the experience of boredom are not identical (Lehr \& Todman, 2009). Boredom results from the combination of boredom proneness and the characteristics of the situation in which an individual is involved: it is perfectly possible that sustained boredom is 
induced in low boredom-prone individuals. If chronic material deprivation, for example, leads people to less externally stimulating situations, boredom may be very high. The latter implies that if one aims to get a grip on socially-induced differences in experienced boredom (and all of its potential negative outcomes regarding subjective well-being), one has to take into account people's individual boredom proneness. So far, however, there is a great paucity of research that studies the relative explanatory power of and interplay between individual differences (such as boredom proneness) and socially-induced differences (such as differences in material deprivation) with respect to aspects of well-being. Indeed, many scholars who study, for example, social differences in (leisure) boredom call for research that takes into account "trait boredom" (Wegner, Flisher, Muller \& Lombard, 2006: 266). Only by doing so researchers will be able to determine to what extent observed social differences in (leisure) boredom are really socially-induced differences, and should therefore be addressed by social policies (rather than individual counselling).

Therefore, besides exploring the potential to measure boredom proneness among young adolescents the second objective of this paper is to assess BPS's explanatory power regarding two indicators that relate to young people's subjective well-being, namely leisure boredom and life satisfaction. Leisure boredom can be considered one specific case of state boredom. Young people have, on average, far more leisure time than adults (Ragheb \& Merydith, 2001) rendering leisure boredom more likely to occur. Moreover, during adolescence, people go through important personal, cognitive, and social developments whilst experiencing increased personal autonomy, the acquisition of different competences, changing relationships with parents, etc. (Hutchinson et al., 2003). Leisure time enables young people to adopt different roles and to experiment with different social identities. In sum, studying leisure boredom among adolescents is important as it may hamper young people's well-being and personal development. 
Satisfaction with life is an individual's overall appraisal of one's life and generally considered the cognitive component of subjective well-being. Life satisfaction has been shown to transcend the immediate effects of mood states and life experiences (Diener et al., 1999) and predicts a wide range of (risky) behaviors and disorders (Proctor, Linley \& Maltby, 2009). Moreover, although life satisfaction is hampered by long-term negative stress and individual problems (e.g., caused by material deprivation, health problems, etc.), in the short run life satisfaction has been shown to (1) be relatively stable and (2) act as a "buffer" against negative stress and the impact of individual problems (Suldo \& Huebner, 2004). For these reasons, life satisfaction is an interesting general outcome to study next to the more specific notion of leisure boredom.

For both leisure boredom and life satisfaction, we explore their relationship with material deprivation - as a proxy for the material environment in which adolescents grow up in - and boredom proneness.

It is known that there is a positive (non-linear) relationship between material deprivation and leisure boredom (Spruyt et al., 2018; Wegner \& Flisher, 2009). In particular, strong material deprivation renders certain leisure activities (including leisure goods such as electronic devices and sports equipment) inaccessible for certain groups (Wegner \& Flisher, 2009). Likewise, more financial resources provide access to a broader range of leisure activities (Granzin \& Haggard, 2000). Previous research revealed that young people from families with less financial resources practice fewer leisure activities and more frequently experience their leisure time as "having nothing to do", unchallenging and monotonous (Harris, 2000). Therefore, we expect materially deprived adolescents to be bored more often during their leisure time. Following the same reasoning - i.e., the idea that limited resources constrain the individual - a negative (possibly non-linear) relationship between material deprivation and life satisfaction is expected.

The key empirical question that we seek to answer is whether these social differences remain after taking into account individual differences in boredom proneness. Different hypotheses can 
be formulated. Starting from the idea that boredom results from the combination of boredom proneness and the situation in which people find themselves, one might expect to find that both BPS and material deprivation have an independent additive effect:

Hypothesis 1a: Even after taking into account individual differences in boredom proneness, indicators of material deprivation are positively related to leisure boredom.

Hypothesis 1b: Even after taking into account individual differences in boredom proneness, indicators of material deprivation are negatively related to life satisfaction.

There are, however, reasons to expect to find an interaction effect. If boredom proneness is people's inclination to be bored, it is reasonable to assume that people who score high on BPS are more sensitive to the amount of arousal in situations. Indeed, one commonly used measure for boredom proneness (the Boredom Susceptibility Scale) is a subscale of Zuckerman's (1979) more encompassing Sensation Seeking Scale. People who score high on this scale are characterized with heightened stimulus seeking and often engage in risky behaviors to satisfy this need. This renders it plausible that BPS moderates the relationship between material deprivation and leisure boredom/life satisfaction so that the relationship between the latter is stronger among people who score high on boredom proneness:

Hypothesis 2a: Boredom proneness moderates the positive relationship between material deprivation and leisure boredom in such a way that material deprivation is more strongly related to leisure boredom among highly boredom prone adolescents.

Hypothesis 2b: Boredom proneness moderates the negative relationship between material deprivation and life satisfaction in such a way that material deprivation is more strongly related to life satisfaction among highly boredom prone adolescents.

The foregoing might apply for boredom proneness in general (Hypothesis 2) but is especially likely for the internal stimulation subdimension of boredom proneness. Indeed, material 
deprivation forces people to be more creative. It is known that the internal stimulation dimension of BPS goes along with a low "need for cognition" (Watt \& Blanchard, 1994) suggesting that boredom prone individuals are less likely to engage in and experience little enjoyment from creative thinking and are less curious. From this, one can derive that when material deprivation increases the odds to live in less stimulating or constraining environments, people who score high on the internal stimulation dimension of BPS may face extra difficulties in compensating for the low arousal of their environment.

\section{Data}

To answer our research questions, we rely on data from the Flemish edition of the third wave of the International Survey of Children's Well-Being (ISCWeB) (data gathered in 2018). The ISCWeB study is an international, inter-cultural and multi-linguistic survey on children's subjective well-being (see http://www.isciweb.org/). Each participating country/region surveyed a large representative sample of at least 1,000 children within each of the targeted age groups, namely children aged 8,10 and 12. In each country a two-stage sample was used in which, first a stratified random sample of primary schools $(N=51)$ was drawn and then within these schools, a random selection of classes was made within the grades where the majority of the children are in the targeted age groups. In Flanders, the sample grid was based on two indicators, namely the educational network (subsidized free vs. public education) and the mother's level of education (schools characterized by a high concentration of pupils with less educated mothers receive in Flanders additional financial resources). Within schools classes in the second (age 8), fourth (age 10) and sixth grade (age 12) were selected.

A response rate of $29 \%$ was achieved at the school level. Schools that refused were replaced by a matching school belonging to the same stratum in the sample grid. The data were recalibrated via post stratification (the weighting coefficients ranged from 0.46 to 1.84 ). 
Surveys were filled out on a tablet in class with the presence of the researcher. The questionnaire consisted of a core module of questions that were asked in every participating country. Each country could add additional questions to this main questionnaire. In Flanders measures related to boredom were added to the questionnaire of young adolescents enrolled in the last grade of primary education. As such, in this study, only the respondents in the age group of 12 years old were selected $(N=1071)$. The research design and protocol received ethical approval from the ethical committee of the university that was responsible for the data collection of the study.

After deleting cases that had a missing value for one of the variables included in our analyses, we arrived at a final sample of 988 respondents. Descriptive statistics are provided in the additional materials which together with all data and syntax code necessary to replicate the results of this paper are available at the Open Science Framework: https://osf.io/wkqzp/?view_only=72b542acdb42466d990a71e6c9a15c3d .

\section{Measures}

In the multilevel regression analyses we take into account a number of control variables that are known to be related to (leisure) boredom (proneness). Regarding to gender $\left(N_{\text {Girls }}=495\right.$, $50.1 \%$ ) it is often found that boys score higher on both boredom proneness and leisure boredom when compared to girls (e.g., Vodanovich \& Kass, 1990; Vodanovich et al., 2005). However, there are also studies that found no relationship (concerning BPS: Biolcati et al., 2018; concerning leisure boredom: Spaeth et al., 2015) or the reverse pattern (concerning boredom in general: Martz et al., 2018).

In Flanders, just like most regions in Europe, the number of people with a migration background has strongly increased in recent decades. This especially applies to the youngest generations. As immigrants are known to strongly differ in terms of material living circumstances, family size, cultural habits, etc., having a migration background was also used as a control variable. 
Respondents were considered to have a migration background if at least one of the parents was not born in Belgium $\left(N_{\text {migration background }}=328 ; 33.2 \%\right)$.

As it is well-known that social contacts are extremely important for young people, satisfaction with friends was also taken into account as a control variable. Satisfaction with friends was measured with four items - I have enough friends; My friends are usually nice to me; My friends and I get on well with each other; My friends support me - judged on a 5-point Likert scale (1: completely disagree -5 : completely agree; Cronbach's alpha $=0.841)$.

In the final step of the analysis, we aim to study the predictive power of boredom proneness and material deprivation regarding leisure boredom and life satisfaction.

Boredom Proneness (internal and external stimulation) was measured by a short version of the Boredom Proneness in Children (BPS-C) scale (Lehr \& Todman, 2009). In this study we used the 10 items of Lehr and Todman's (2009) BPS-C scale that are also part of the Boredom Proneness Short Form (BPS-SF) scale (Vodanovich, Wallace \& Kass, 2005). BPS-Internal stimulation (i.e., It's easy for me to play by myself) and BPS-External stimulation (i.e., I'm only really happy if things around me don't stay the same for very long) were measured by five items each. All items were rated on a 5-point Likert scale (1: strongly disagree -5 : strongly agree) Results of the scale analysis are presented in the Results section.

Research that studied the empirical relationship between young people's economic position and aspects of subjective well-being shows that, rather than SES or parental income, children's own views on their living circumstances and financial situation is strongly and positively related to subjective well-being and/or life satisfaction (Main \& Bradshaw, 2012). Therefore, in constructing a measure for material deprivation we started from a child-derived measure composed of three indicators: (1) satisfaction with their material living conditions (0-10), (2) whether the child worried about their family's money (never, sometimes, often, always, don't know), and (3) whether the child had enough food (never, sometimes not, most of the time, 
always, don't know). A categorical principal component analysis (CATPCA) revealed one dimension with acceptable reliability (Cronbach's alpha=0.608). Because the distribution of the raw object scores of this measure was rather skewed (range: -0.25 to 25.13 ), we used a transformed 20 decile interval scale.

To measure leisure boredom, we used four items of the boredom subscale of the leisure experiences scale developed by Barnett (2005) - For me, free time, just drags on and on; Free time is boring; In my free time I find it difficult to find something pleasant to do; During my free time I almost always have something to do [reverse coded] (Cronbach's alpha= 0.680) that were rated on a 5-point Likert scale (1: strongly disagree - 5: strongly agree). The items refer to an individual's overall assessment of their leisure time. As such they are assumed to be the result of both individual (e.g., boredom proneness) and social (e.g., material deprivation) factors. We reverse coded the items and calculated an averaged scale so that higher scores reflected more leisure boredom. Life satisfaction was measured with four items of the Student Life Satisfaction Scale (SLSS) (i.e., My life is going well; My life is just right; I have a good life; I have what I want in life) (Huebner, 1991). The ISCWeB consortium added a fifth item (i.e., The things in my life are excellent) adapted from the Subjective Well-Being Scale (SWLS) (Diener, Emmons, Larsen \& Griffin, 1985). All items were rated on an 11-point Likert scale (0: completely disagree - 10: completely agree) (Cronbach's alpha=0.942).

\section{Analysis}

First, Confirmatory Factor Analysis (CFA) as implemented by Stata 14.0 was used to test the multidimensional nature of the BPS-C scale. The goodness-of-fit of the models was evaluated by fit indices such as the $\chi^{2}$ statistic, root mean square error of approximation (RMSEA), comparative fit index (CFI), and Tucker-Lewis fit index (TLI). In a second step, we assessed the relationship between boredom proneness and material deprivation with leisure boredom and 
life satisfaction. Due to the clustered nature of our data - respondents were surveyed in schools - we used multilevel models to estimate robust standard errors.

\section{Results}

\section{Scale analysis of the BPS-C scale}

Table 1 summarizes the results for the CFA analyses. A baseline measurement model with two factors (i.e., internal vs. external stimulation) and no error covariances was tested for the twodimensional structure of the BPS-C internal and external stimulation scales. Results of the CFA and fit indices suggested that the measurement model could be improved by incorporating (1) a cross-loading (Model 2) of one item belonging to the external stimulation subscale 'I think most of the things I have to do are too easy for me' to the internal stimulation scale and (2) an error covariance (Model 3) in the external stimulation scale between 'I'm only really happy if things around me don't stay the same for very long' and 'Unless I am doing something really exciting, or even dangerous, Ifeel bored'. Specifying these adaptations was appropriate given that the internal and external stimulation items refer to different aspects of a more general underlying phenomenon, i.e. people's boredom proneness.

\section{[Table 1]}

In line with other research (Watt \& Blanchard, 1994), we found that boys $(M=0.082)$ scored higher than girls $(M=-0.104)$ on the external stimulation subscale $(E t a=0.196 ; p=0.000)$. No gender differences were found for internal stimulation. For migration status, we found a small but significant difference for internal stimulation $(E t a=0.065 ; p=0.040)$ with migrants $(M=-$ $0.032)$ scoring slightly lower when compared to their non-migrant peers $(M=0.008)$. Material deprivation correlated positively but weakly with both internal and external stimulation ( $r=$ 0.062 and $r=0.094$ respectively; $p<0.050$ ).

\section{The relationship between BPS-C and Leisure Boredom and Life Satisfaction}


To further explore the usefulness of the BPS-C scales we assessed their predictive power with regard to potential outcomes that relate to subjective well-being. As outcome variables we studied leisure boredom and life satisfaction. For each outcome, five models were estimated. In Model 1 gender, migration background and the level of material deprivation was entered. In Model 2 model we entered a quadratic term for material deprivation to test whether the relationship between material deprivation and the outcomes was non-linear. In Model 3 we added the two boredom proneness scales. Because it is well known that friends are very important in adolescence, we also added satisfaction with people's social life (in particular friends) as an additional control variable (for life satisfaction we also controlled for leisure boredom as a robustness check). In the next two models we tested interaction terms between the boredom proneness measures and levels of deprivation. This way we could assess whether people who are more predisposed to boredom react differently to their living circumstances (measured in terms of material deprivation).

\section{Leisure boredom}

Our models show no gender differences in leisure boredom (Table 2). We did find that young adolescents with a migration background are also more bored. This difference is already apparent in Model 1, but remained relatively stable when control variables were entered in subsequent models. It is important to stress that this effect is 'net' of the level of child-reported material deprivation. As expected, child-reported material deprivation predicted leisure boredom. Model 2 shows that the relationship was non-linear, suggesting that especially among young people who live in highly materially deprived circumstances, material deprivation was quite strongly related to more leisure boredom. A very similar relationship was found for overall life satisfaction suggesting that also among adolescents living in the wealthiest circumstances life satisfaction is lower and leisure boredom somewhat higher. We come back to this observation in the discussion section. 


\section{[Table 2]}

In Model 3, we added the two subscales of boredom proneness. Both indicators were positively related to leisure boredom. People who were more prone to boredom were more bored during their leisure time. Interestingly, the strength of the effect varied with the effect of external stimulation (i.e., people's need for external stimulation), becoming more than twice as strong when compared to the internal stimulation scale (i.e., people's capacity to entertain themselves).

In Models 4 and 5, we tested interaction terms. As the 'main' effect of child-reported material deprivation was nonlinear (Model 2), we followed Buis (2011) who recommended that in order to test an interaction effect one needs to specify interaction terms with both the singular and quadratic term of child-reported material deprivation. For leisure boredom, no interaction effects were found, suggesting that the effects of child-reported material deprivation and boredom proneness are additive.

\section{Life Satisfaction}

For life satisfaction we found a significant relationship with gender (Model 1, Table 3), indicating that boys were more satisfied with their lives than girls. In none of the models, was a difference found for having a migration background.

Model 1 shows a substantial and significant negative effect of child-reported material deprivation on life satisfaction which in Model 2 turned out to be non-linear. The pattern is very similar to the one observed for leisure boredom: especially among young adolescents who felt more materially deprived, the detrimental effect of material deprivation on life satisfaction was substantial. That said, and as indicated before, also among youth with the least material deprivation we found somewhat lower scores for life satisfaction.

Also, for life satisfaction, we found a significant relationship with both subscales of boredom proneness but again the strength of the effect parameters varied (Model 3). For life satisfaction, 
internal stimulation seemed to be more important when compared to external stimulation (for leisure boredom we observed the opposite pattern).

[Table 3]

We found a significant interaction pattern between child-reported material deprivation and the internal stimulation subscale of boredom proneness (Model 5). As Figure 1 shows the relationship between child-reported material deprivation and life satisfaction was much stronger for young people who scored high ( +1 standard deviation) on internal stimulation. This suggests that among adolescents who are more prone to boredom - and more specifically who are in need of more internal stimulation - the relationship with child-reported material deprivation is much stronger. Interestingly, we observed this pattern both in terms of the positive effect among adolescents who live in less deprived situations, as well as of the negative effect among adolescents who live in more materially deprived situations. So, with respect to life satisfaction, it is clear that the internal stimulation dimension of BPS-C renders adolescents not only more likely to be less satisfied with their life, but also more sensitive to the perceived material conditions of their living circumstances. The same interaction effect can also be interpreted in reverse. Indeed, from Figure 1 one can also derive that among the most materially deprived the impact of people's boredom proneness (here: the need for internal stimulation) is the largest: when material means are lacking, individual differences have free play.

\section{[Figure 1]}

\section{Discussion and conclusion}

Although boredom may be detrimental for young adolescents' psychological development, so far little research has studied boredom proneness and its correlates among young adolescents (but see Lehr \& Todman, 2009). This gap deserves attention for two reasons. First, as long as one cannot take into account individuals' general disposition to be bored, it remains unclear to 
what extent social differences in leisure boredom and life satisfaction, amongst others, are genuine socially-induced differences that can and should be addressed with social policies. Second, research on boredom proneness has repeatedly revealed at least two subdimensions. Differences in scores on the internal versus external subdimension of BPS may reflect very different reasons for the occurrence of boredom (and its associated negative effects in terms of well-being) and in this way guide different intervention strategies.

Therefore, this paper studied boredom proneness in a large probability sample of young adolescents. It aimed to bring together two bodies of literature that until now have largely developed separately from each other. Whereas scholars who study BPS from a psychological perspective have invested much in scale development and assessing the relationship between BPS and a wide range of outcomes (including many psychological disorders), such research left the question as to how widespread boredom proneness is in the population at large unanswered. Sociologists and scholars who relied on representative samples often had to work with one-item measures. More importantly, they lacked good measures for people's dispositional tendency for boredom, rendering it difficult to judge the real significance of people's living environment with respect to boredom.

Our study is among the first that has implemented the BPS-C measure in a representative survey among young adolescents. Although we found for both the internal and external stimulation BPS-C scales significant and theoretically consistent relationships with relevant outcomes, their mutual correlation was very low and non-significant. At this point our results differ from those of Lehr and Todman (2009) who found that a combined BPS-C which does not distinguish between internal and external stimulation (a practice which is quite common in the BPSliterature) performed well. More research is needed to assess whether our findings can be replicated in a different context. 
At the most general level, our findings indicate that both individual and social factors predict leisure boredom and life satisfaction. First, even after taking into account individual differences in boredom proneness and other characteristics, we found that child-reported material deprivation was associated with more leisure boredom and lower life satisfaction (accept hypotheses 1a \& 1b). In that context, scholars increasingly warn against interpreting such a net relationship as a strictly individual problem. Studies have indeed repeatedly shown that for young people spending leisure time in organized contexts is socio-economically and culturally structured (see Eccles, Barber, Stone \& Hunt, 2003; Mahoney, 2000). Research shows that leisure is among the first expenditures which households facing economic difficulties cut back on (Deutsch, Guio, Pomati \& Silber, 2015). Moreover, Roets et al. (2015) found that a lack of material and financial resources clearly influences the ways in which children can shape and give meaning to their regimes of time. So, taking the finding that, even after controlling for different factors, material deprivation predicts leisure boredom and lowers life satisfaction into a practical context, the preceding research suggests that to achieve an accessible supply of leisure activities for all, removing financial barriers is important. This, however, will only be successful when it is embedded in a more encompassing approach that establishes a better fit with young people's living environment. At the same time the non-linear pattern indicated that also at the other end of the deprivation continuum - among youth who are least materially deprived - leisure boredom was somewhat higher and life satisfaction somewhat lower compared to youth who scored more lower-middle range on the deprivation measure. This was an unanticipated finding which, however, does align with a growing body of research that focuses on the so called 'costs of privilege' (Coren \& Luthar, 2014) that result from high pressure to perform (in school, leisure, etc.). Adolescents whose privileged parents focus too strongly on achievement may strive solely for extrinsic rather than intrinsic goals and ultimately 
develop narcissistic exhibitionism and all associated problem behavior (e.g., substance abuse and adjustment problems) (Luthar \& Barkin, 2012).

Second, we found that for life satisfaction the BPS-C measures had a significant relationship even after taking into account leisure boredom. This observation has two, albeit related, implications. It strongly suggests that boredom proneness must also predict (1) other outcomes that (2) potentially mediate the negative effects of BPS on life satisfaction. Or formulated differently: boredom proneness most likely does more than just predict the occurrence of boredom. This conclusion aligns with the fact that some scholars consider boredom proneness one of the subdimensions of sensation seeking (see Vodanovich, 2003).

This study is not without limitations. First, although the results based on our cross-sectional data are promising, further longitudinal research should assess (1) the within-subject stability of young people's tendency to be bored when assessed with the measure that we used here and (2) the causal impact of boredom proneness on different aspects of young people's lives. Moreover, to further deepen our understanding of boredom proneness, future research should study it simultaneously with other dispositions so that it becomes possible to assess their unique contribution to the phenomena at hand. Second, in this paper we relied on a rather traditional unidimensional notion of material deprivation by limiting it to the strictly perceived financial situation of young adolescents. In line with recent advances in the literature on the measurement of material deprivation (e.g., Bastos \& Machdo, 2009), multidimensional measures (including non-monetary aspects of deprivation that refer to social interactions and cognitive development) could refine the picture concerning how exactly, that is, along which pathways, material deprivation decreases life satisfaction and increases leisure boredom.

The former considerations, however, do not undermine the fundamental message of this paper, namely that there is much to gain by attempts to further unravel the relationships between dispositions and social conditions when we attempt to understand aspects of young people's 
well-being. Indeed, our results did not only show that both psychological dispositions (i.e., BPS) and (child-reported) material living conditions (i.e., child-reported material deprivation) predict outcomes such as leisure boredom and life satisfaction. For life satisfaction, we also found a significant interaction between dispositions and living conditions (accept hypothesis 2b; no support for hypothesis 2a). And, more specifically, we found that scoring high on internal stimulation - i.e., people who find it difficult to keep oneself interested and entertained renders young adolescents not only more likely to be less satisfied with their lives but also more sensitive to the perceived material conditions of their living conditions. From a policy perspective, this suggest that young adolescents who live in socially deprived conditions would benefit most from successful attempts to enhance people's internal stimulation capacity. Although boredom proneness is generally considered a trait or a disposition, and thus by definition characterized by a strong inertia, this does not imply that it cannot be influenced by activities that are specifically designed to increase an individuals' psychological capacities (e.g., mindfulness, etc.). The strength of our finding is that it acknowledges the detrimental consequences of material deprivation (and hence underscores the importance of lowering material and financial barriers) and at the same time stimulates further research that assesses potential determinants of more psychological traits and mechanisms (as well as more applied research concerning strategies to impact upon these). 


\section{References}

Barnett, L.A. (2005). Measuring the ABCs of leisure experience: awareness, boredom, challenge, distress. Leisure Sciences, 27, 131-155.

Bastos, A. \& Machado, C. (2009) Child poverty: a multidimensional measurement. International Journal of Social Economics, 36, 237-251.

Biolcati, R., Mancini, G., \& Trombini, E. (2018). Proneness to boredom and risk behaviors during adolescents' free time. Psychological Reports, 121, 303-323.

Buis, M. (2011). Stata tip \#: Exploring model consequences by plotting different predictions. Retrieved April 28, 2019, from $\underline{\text { http://www.maartenbuis.nl/wp/inter_quadr/inter_quadr.html }}$

Coren, S.A., \& Luthar, S.S. (2014) Pursuing perfection: distress and interpersonal functioning among adolescents boys in single-sex and co-educational independent schools. Psychology in the Schools. 51, 931-946.

Deutsch, J., Guio, A.-C., Pomati, M., \& Silber, J. (2015). Material deprivation in Europe: which expenditures are curtailed first? Social Indicators Research, 120, 723-740.

Diener, E., Emmons, R., Larsen, J., \& Griffin, S. (1985). The satisfaction with life scale. Journal of Personality Assessment, 49, 71-75.

Eastwood, J.D., Frischen, A., Fenske, M.J., \& Smilek, D. (2012). The unengaged mind: defining boredom in terms of attention. Perspectives on Psychological Science, 7, 482495.

Eccles, J.S., Barber, B.L., Stone, M., \& Hunt, J. (2003). Extracurricular activities and adolescent development. Journal of Social Issues, 59(4), 865-889. 
Farmer, R.F., \& Sundberg, N.D. (1986). Boredom proneness - the development and correlates of a new scale. Journal of Personality Assessment, 50, 4-17.

Goldberg, Y.K., Eastwood, J.D., Laguardia, J., \& Danckert, J. (2011). Boredom: an emotional experience distinct from apathy, anhedonia, or depression. Journal of Social and Clinical Psychology, 6), 647-666.

Granzin, K.L., \& Haggard, L.M. (2000). An integrative explanation for quality of life: development and test of a structural model. In E. Diener \& D.R. Rahtz (Eds.), Advances in quality of life theory and research (pp. 31-63). Dordrecht: Kluwer Academic Publishers.

Harris, M.B. (2000). Correlates and characteristics of boredom proneness and boredom. Journal of Applied Social Psychology, 30, 576-598.

Huebner, E.S. (1991). Initial development of the student's life satisfaction scale. School Psychology International, 12, 231-240.

Hutchinson, S.L., Baldwin, C.K., \& Caldwell, L.L. (2003). Differentiating parent practices related to adolescent behavior in the free time context. Journal of Leisure Research, 35, $396-422$.

Lehr, E., \& Todman, M. (2009). Boredom and boredom proneness in children: implications for academic and social adjustment. In M. Todman (Ed.), Self-regulation and social competence: psychological studies in identity, achievement and work-family dynamics (pp. 79-90). Athens: ATINER Press.

Luthar, S.S., \& Barkin, S.H. (2012). Are affluent youth truly 'at risk'? Vulnerability and resilience across three diverse samples. Development and Psychopathology, 24, 429-449.

Mahoney, J. (2000). School extracurricular activity participation as a moderator in the development of antisocial patterns. Child Development, 71, 502-516. 
Main, G., \& Bradshaw, J. (2012). A Child Material Deprivation Index. Child Indicators Research, 5, 503-521.

Martz, M.E., Schulenberg, J.E., Patrick, M.E., \& Kloska, D.D. (2018). “I am so bored!”: prevalence rates and sociodemographic and contextual correlates of high boredom among American adolescents. Youth \& Society, 50, 688-710.

Pekrun, R., Hall, N.C., Goetz, T., \& Perry, R.P. (2014). Boredom and academic achievement: Testing a model of reciprocal causation. Journal of Educational Psychology, 106, 696710.

Proctor, C.L., Linley, P.A., \& Maltby, J. (2009). Youth life satisfaction: A review of the literature. Journal of Happiness Studies, 10, 583-630.

Ragheb, M.G., \& Merydith, S.P. (2001). Development and validation of a multidimensional scale measuring free time boredom. Leisure Studies, 20, 41-59.

Roets, G., Cardoen, D., Bie, M.B.-D., \& Roose, R. (2015). We make the road by walking: challenging conceptualisations of leisure time for children in poverty. Children \& Society, 29, 277-287.

Sommers, J., \& Vodanovich, S.J. (2000). Boredom proneness: its relationship to psychologicaland physical-health symptoms. Journal of Clinical Psychology, 56, 149-155.

Spaeth, M., Weichod, K., \& Silbereisen, R.K. (2015). The development of leisure boredom in early adolescence: predictors and longitudinal associations with delinquency and depression. Developmental Psychology, 51, 1380-1394.

Spruyt, B., Vandenbossche, L., Keppens, G., Siongers, J., \& Van Droogenbroeck, F. (2018). Social differences in leisure boredom and its consequences for life satisfaction Aamong young people. Child Indicators Research, 11, 225-243. 
Suldo, S.M., \& Huebner, E.S. (2004). Does life satisfaction moderate the effects of stressful life events on psychopathological behavior during adolescence? School Psychology Quarterly, 19, 93-105.

Vodanovich, S.J. (2003). Psychometric measures of boredom: a review of the literature. The Journal of Psychology, 137, 569-595.

Vodanovich, S.J., \& Kass, S.J. (1990). Age and gender differences in boredom proneness. Journal of Social Behavior and Personality, 5, 297-307.

Vodanovich, S.J., Wallace, C.J., \& Kass, S.J. (2005). A confirmatory approach to the factor structure of the Boredom Proneness Scale: evidence for a two-factor short from. Journal of Personality Assessment, 85, 295-303.

Vodanovich, S.J., \& Watt, J.D. (2016). Self-report measures of boredom: an updated review of the literature. The Journal of Psychology, 150, 196-228.

Watt, J.D., \& Blanchard, M. (1994). Boredom proneness and the need for cognition. Journal of Research in Personality, 28, 44-51.

Watt, J.D., \& Vodanovich, S.J. (1999). Boredom proneness and psychosocial development. The Journal of Psychology, 133, 303-314.

Wegner, L., \& Flisher, A.J. (2009). Leisure boredom and adolescent risk behaviour: a systematic literature review. Journal of Child \& Adolescent Mental Health, 21, 1-28.

Wegner, L., Flisher, A.J., Muller, M., \& Lombard, C. (2006). Leisure boredom and substance use among high school students in South Africa, Journal of Leisure Research, 38, 249266.

Zuckerman, M. (1979). Sensation seeking: Beyond the optimal level of arousal. Hillsdale: Erlbaum. 
